\title{
Pelargonium zonate spot virus Is Transmitted Vertically via Seed and Pollen in Tomato
}

\author{
M. Lapidot, D. Guenoune-Gelbart, D. Leibman, V. Holdengreber, M. Davidovitz, \\ Z. Machbash, S. Klieman-Shoval, S. Cohen, and A. Gal-On
}

First, second, sixth, and eight authors: Department of Vegetable Research; third, fourth, and ninth authors: Department of Plant Pathology; and fifth author: Department of Entomology, Volcani Center, ARO, Bet Dagan 50250, Israel; and seventh author: Hazera Genetics, Mivhor, Mobile P. Lakhish Darom 79134, Israel.

Accepted for publication 12 April 2010.

\begin{abstract}
Lapidot, M., Guenoune-Gelbart, D., Leibman, D., Holdengreber, V., Davidovitz, M., Machbash, Z., Klieman-Shoval, S., Cohen, S., and Gal-On, A. 2010. Pelargonium zonate spot virus is transmitted vertically via seed and pollen in tomato. Phytopathology 100:798-804.

In autumn 2007, a new disease with unknown etiology was observed in open-field tomato (Solanum lycopersicum) in the Lachish region of Israel. The symptoms included mild mosaic, leaf malformation, and severe stunting of the plants. The causal agent was readily transmitted mechanically from the sap of infected plants to indicator plants. Viral particles were purified from infected plants and cDNA was synthesized from RNA isolated from the particles. Cloning and sequencing of the

cDNA showed $95 \%$ identity to RNA 3 of Pelargonium zonate spot virus (PZSV). Using reverse-transcription polymerase chain reaction, PZSV was detected in both seed and pollen grains of infected tomato plants. Attempts to disinfect seed by using hydrochloric acid and trisodium phosphate failed to eliminate this PZSV detection. Seed from infected tomato plants gave rise to infected seedlings with a seed-transmission rate of PZSV of 11 to $29 \%$. Pollen grains collected from flowers of infected plants were used to hand pollinate healthy mother tomato plants. Although none of the pollinated mother plants became infected with PZSV, 29\% of the seedlings produced from seed harvested from these plants were found to be infected. This is the first demonstration that PZSV is transmitted vertically via both pollen and seed in tomato plants.
\end{abstract}

Pelargonium zonate spot virus (PZSV) belongs to the family Bromoviridae and was recently assigned to the new genus Anulavirus (6). The virus has a $25-$ to $35-\mathrm{nm}$ diameter quasispherical particle with a genome composed of three single-stranded RNA segments that encode four proteins. RNA-1 (3,383 nucleotides ([nt]) and RNA-2 (2,435 nt) are monocistronic and encode nonstructural proteins involved in viral replication. RNA-3 (2,659 nt) is bicistronic and encodes the viral movement (open reading frame [ORF] 3a) and capsid (ORF 3b) proteins (MP and CP, respectively) (4). PZSV was originally isolated from Pelargonium zonale plants (18) but, in 1980, was found to be the causal agent of a disease in tomato (Solanum lycopersicum L.) in southern Italy (5). PZSV induces severe disease symptoms in tomato plants, characterized by chlorotic and necrotic ring and line patterns on the leaves and fruit together with plant stunting, leaf malformation, and reduced fruit set, which often result in plant death (5). Nearly 20 years after the report from Italy, the virus has been detected in tomato plants in Spain (14) and France (7) and, recently, the virus was detected in tomato fields in California (United States) $(9,13)$. The sequence variation among the different isolates is not known, because only the genome of the Italian isolate has been fully sequenced (4). The Spanish and French isolates of the virus were identified based on serology, whereas the American isolate has been partially (ORF 3b) sequenced $(7,13,14)$. Recently, a high incidence of PZSV has been reported in greenhouse tomato plants in northeastern Spain (3). The virus

Corresponding author: M. Lapidot; E-mail address: lapidotm@ volcani.agri.gov.il

*The $e$-Xtra logo stands for "electronic extra" and indicates that Figure 1 appears in color online.

doi:10.1094/PHYTO-100-8-0798

(c) 2010 The American Phytopathological Society also infected pepper plants that were in the tomato greenhouses. A partial 697-nt fragment (ORF 3a) of RNA 3 was cloned and sequenced from the PZSV-infected pepper and tomato plants. Sequences representing the viral ORF $3 \mathrm{a}$ from both infected plants were found to be identical (3).

The virus is readily transmitted mechanically from the sap of infected plants and has been shown to be seed transmitted in Nicotiana glutinosa L., with an efficiency of $\approx 5 \%$ (5). Pollen from systemically infected $N$. glutinosa plants has also been shown to infect plants when mechanically inoculated onto leaves of test plants (5). Although Vovlas et al. (20) found pollen collected from naturally infected tomato plants to be contaminated with PZSV, the virus was not found to be transmitted via tomato seed. While looking for alternative hosts, these authors found that Diplotaxis erucoides (L.) DC., an endemic weed in southern Italy, serves as a symptomless host for the virus. They also found that PZSV is seed transmitted in D. erucoides with an efficiency of $\approx 5 \%$. When pollen from infected $D$. erucoides was rubbed gently onto leaves of tomato plants, 8 of 10 plants became infected within 10 days (20). These authors also presented preliminary results showing that a thrips species (Melanothrips fuscus) feeding on flowers of $D$. erucoides carries pollen grains on its body. When these thrips were transferred to tomato seedlings, 1 of 10 plants showed PZSV symptoms 25 days later (20). Hence, it was suggested that PZSV might be transmitted by thrips in an unusual manner. Nevertheless, the possibility of accidental mechanical inoculation due to insect and plant handling during transfer from infected to test plants could not be ruled out.

In autumn 2007, a new disease with virus-like symptoms but of unknown etiology was observed in open-field tomato crops in the Lachish region of Israel. The symptoms included a light mosaic, leaf malformation, and severe stunting of the plants. The causal agent was mechanically transmitted to indicator plants and, after RNA isolation and cDNA synthesis, was identified as PZSV 
through partial sequencing. The objective of this article was to characterize this new-to-Israel tomato-infecting virus and to determine its natural mode of transmission.

\section{MATERIALS AND METHODS}

Sample collection. In October 2007, cuttings of symptomatic tomato plants exhibiting symptoms of mild mosaic, leaf malformation, and severe stunting were collected from a field in Lachish and maintained in a research greenhouse for subsequent testing. These plants were used for total RNA extraction and as initial source plants for subsequent sap transmission experiments.

Electron microscopy. PZSV particles from leaf tissue of symptomatic tomato plants were observed by electron microscopy. Formvar carbon-coated grids were incubated on droplets of crude leaf extract at room temperature for $2 \mathrm{~min}$, washed with water, and negatively stained with $2 \%$ uranyl acetate. Images were taken by transmission electron microscope (TEM) (Tecnai G2; FEIPhilips, Eindhoven, The Netherlands).

Viral isolation, cloning, and sequencing. To identify the virus, virions were purified from $30 \mathrm{~g}$ of infected $N$. benthamiana Domin. leaves 3 weeks after inoculation, as described by Roossinck and White (18) for Cucumber mosaic virus purification. Viral RNA was isolated from the purified virions as follows: after incubating the purified virus in $0.5 \%$ sodium dodecyl sulfate for $5 \mathrm{~min}$, RNA was extracted with phenol/chloroform $(1: 1$, $\mathrm{vol} / \mathrm{vol}$ ) and precipitated in ethanol. cDNA was synthesized with $0.1 \mathrm{mg}$ of purified viral RNA as a template using the Universal RiboClone cDNA Synthesis System from Promega Corp. (Madison, WI) with random hexamers as primers.

The entire PZSV genome (RNAs 1, 2, and 3) was amplified by reverse-transcription polymerase chain reaction (RT-PCR) using a Verso cDNA Kit (ABgene, Epsom, UK) according to the manufacturer's instructions, with specific primers based on the sequence obtained earlier and the Italian PZSV strain sequence (accession nos. AJ272327, AJ272328, and AJ272329). The following primers were used: for RNA 1, forward primer PZSV-1F (5'GGTTTGAGTGCATTTTGTGTATTTGG-3', nucleotides 1 to 26 of RNA 1), reverse primer PZSV-2635R (5'-CTTTTTGGTCT CTCTTAGAGAAAC-3', nucleotides 3383 to 3360 of RNA 1); for RNA 2, forward primer PZSV-1F (5'-GGTTTGAGTGCAT TTTGTGTATTTGGTTCA-3', nucleotides 1 to 30 of RNA 2), reverse primer PZSVR2-2435R (5'-TGGTCTCTCTTAGAGA AACC-3', nucleotides 2435 to 2416 of RNA 2); and for RNA 3, forward primer PZSVR3-1F (5'-GTTTGAACTTAGTAATTGC ATGTGT-3', nucleotides 1 to 25 of RNA 3), reverse primer PZSV-2635R (5'-CTTTTTGGTCTCTCTTAGAGAAAC-3', nucleotides 2659 to 2635 of RNA 3). According to the reported sequence of the Italian PZSV isolate, the 5' first 60 nt of RNA 1 and RNA 2 are identical and the $3^{\prime}$ last 42 nt of RNA 1 and RNA 3 are identical. Hence, the same forward primer (PZSV-1F) was used for cloning of both RNA 1 and RNA 2 and the same reverse primer (PZSV-2635R) was used to clone RNA 1 and RNA 3.

Host range. To determine the host range for PZSV, leaves of symptomatic tomato and $N$. glutinosa plants showing clear disease symptoms 2 weeks after inoculation were used to sap inoculate the first true leaf of test plants using carborundum as an abrasive (11). Five plants per each test plant species were inoculated and each experiment was repeated three times. Plants were grown in a greenhouse and inspected daily for symptom appearance for up to 4 weeks after inoculation. Infectivity was determined by back inoculation to $N$. glutinosa test plants. Samples were taken from the plant apex once symptoms appeared or, if no symptoms appeared, then at 4 weeks postinoculation for back inoculation as well as for testing by RT-PCR.

Viral detection by RT-PCR (in plant, seed, and pollen). Total RNA was extracted from plant tissue, seed, or pollen grains.
From plant tissue, total RNA from $100 \mathrm{mg}$ of fresh leaves was extracted using TRI-reagent (Sigma-Aldrich, St. Louis) according to the manufacturer's instructions. From tomato seed, total RNA from infected (with and without disinfection treatments) and healthy tomato seed was extracted after soaking the seed in phosphate-buffered saline buffer for $2 \mathrm{~h}$. The seed were then frozen with liquid nitrogen and crushed using a mortar and pestle, and total RNA was extracted using the QIAMP Viral RNA Mini Kit (Qiagen, Hilden, Germany) according to the manufacturer's instructions. From pollen grains, total RNA extraction from infected and healthy tomato pollen grains was performed according to Levin and Gilboa (12). The nucleic acid concentrations from the different extractions were measured using a spectrophotometer (ND-1000; NanoDrop, Wilmington, DE).

RT-PCR analysis was performed to detect PZSV accumulation in plants, seed, and pollen. The first-strand cDNA was synthesized using $1 \mu \mathrm{g}$ of total RNA extract using complementary primers specific to PZSV with a Revertaid First-Strand cDNA Synthesis Kit (Fermentas, Ontario, Canada) according to the manufacturer's instructions. cDNA $(1 \mu \mathrm{l})$ was used as a template for standard PCR. cDNA was denatured at $94^{\circ} \mathrm{C}$ for $5 \mathrm{~min}$; followed by 30 cycles of $94^{\circ} \mathrm{C}$ for $30 \mathrm{~s}, 56^{\circ} \mathrm{C}$ for $30 \mathrm{~s}$, and $72^{\circ} \mathrm{C}$ for $1 \mathrm{~min}$; followed by $10 \mathrm{~min}$ at $72^{\circ} \mathrm{C}$. The primers used for PZSV RNA 3 detection were as follows: for cDNA synthesis, reverse primer PZSV-2635R; for PCR, forward primer PZSVR3-1239F (5'CATCGACGCAGTCTGCCTT-3', nucleotides 1239 to 1257 of RNA 3) and reverse primer PZSVR3-2133R (5'AGTCCGTAGCAAACAGATACA-3', nucleotides 2153 to 2133 of RNA 3), amplifying a 894-nt fragment of PZSV RNA3. The resulting fragment was cloned into a pTZ57R plasmid suitable for T-A cloning and used as a positive control.

Seed harvesting and disinfection treatments. Seed were harvested from PZSV-infected tomato plants (cvs. M82 and R13). The plants were mechanically inoculated (at the two-leaf stage) with the virus and grown to fruit maturity in the greenhouse. Seed were harvested from the ripened tomato fruit as follows. Fruit were cut in half and all of the seed were removed, transferred to a small container, and left to ferment for $24 \mathrm{~h}$ at $27^{\circ} \mathrm{C}$. Seed were then transferred to a sieve, washed with water, and allowed to dry at room temperature. The seed of each fruit were stored at room temperature in separate bags until use.

Seed-disinfection treatments included the following: incubating in a $2 \%(\mathrm{vol} / \mathrm{vol}) \mathrm{HCl}$ solution for $2 \mathrm{~h}$, then incubating in $10 \%$ (wt/vol) trisodium phosphate (TSP) for $3 \mathrm{~h}$ or incubating in bleach ( $10 \%$ vol/vol sodium hypochlorite) for $3 \mathrm{~h}$. Following each treatment, the seed were rinsed in water for $30 \mathrm{~min}$, then dried and stored at room temperature.

Plant pollination. Twenty clean tomato "mother" plants (cv. M82) were grown from seed in isolation in a greenhouse. The plants were tested by RT-PCR to verify no infection with PZSV. Once the plants started to flower, flowers were manually emasculated and pollinated with pollen collected from PZSV-infected plants grown in a separate growth chamber. Once the mother plants bore fruit, seed were extracted from each individual fruit, treated with $\mathrm{HCl}$ and $\mathrm{TSP}$, and stored separately at room temperature.

Seed transmission. Seed extracted from PZSV-infected and control noninoculated plants were sown in sterile soil in Todd planter flats (Speedling Inc., Sun City, FL) and were grown for a month in isolation in a greenhouse. The emerging seedlings were evaluated daily for the development of PZSV-induced disease symptoms. After 4 weeks, the seedlings were tested for the presence of PZSV by RT-PCR and by inoculation to $N$. glutinosa test plants. In the first two experiments, only the symptomatic plants were also found positive by RT-PCR and by inoculation to test plants. Hence, in the following experiments, only the symptomatic plants were also tested by RT-PCR and by inoculation to test plants. 
Soil transmission. PZSV-infected $N$. glutinosa plants were grown for nearly 2 months in pots containing Lava soil grade $0-4$, and then removed while leaving their infected roots in the soil. Then, 14-day-old (first-true-leaf stage) tomato seedlings were transplanted into the contaminated soil and allowed to grow for another 2 months. The transplanted tomato plants were monitored for the appearance of PZSV-induced symptoms and tested for the presence of PZSV by RT-PCR and by inoculation to $N$. glutinosa test plants.

\section{RESULTS}

Identification, cloning, and sequencing of PZSV. A new disease was observed in open-field tomato in autumn 2007. Symptomatic tomato plants were collected from the field and maintained in a research greenhouse. The causal agent was mechanically transmitted to tomato test plants where symptoms were reproduced, as well as to several indicator species (Fig. 1). Crude extracts of leaf tissue from symptomatic tomato plants were negatively stained and analyzed by TEM, revealing spherical particles, 25 to $30 \mathrm{~nm}$ in diameter (not shown). Viral particles were purified from infected $N$. benthamiana plants and RNA was isolated from them and used as a template to synthesize cDNA using random hexamers as primers. Cloning and sequencing of a 900-nt cDNA showed a high identity level to RNA 3 of the Italian isolate of PZSV (accession no. NC003651) (not shown). To clone the full-length RNA 3, another RT-PCR was performed, using specific primers based on the Italian PZSV sequence. The cloned and sequenced 2,659-nt cDNA showed 95\% identity to RNA 3 of the Italian isolate of PZSV (not shown). RNA 3 contained the ORFs coding for the CP and MP of PZSV. Indeed, the Israeli isolate MP and $\mathrm{CP}$ showed high amino acid sequence identity to the Italian isolate ORF $3 \mathrm{a}$ and $3 \mathrm{~b}$, respectively (Table 1). Further cloning and sequencing of RNAs 1 and 2 showed $92 \%$ identity to RNA 1 of the Italian isolate and $98 \%$ identity to RNA 2 of the Italian isolate, respectively. Both ORFs $1 \mathrm{a}$ and $2 \mathrm{a}$ were found to have a high amino acid identity to the Italian isolate (Table 1). Thus, we confirmed that the virus isolated from tomato plants in Lachish, Israel, is indeed an isolate of PZSV.

Host range. Indicator plants were mechanically inoculated with PZSV in the greenhouse, using sap from infected N. glutinosa plants (Fig. 1). Based on their reaction to the virus, the inoculated plants could be divided into three groups: plants with localized infection (symptoms appeared only on the inoculated leaf but the virus did not spread to the plant apex), plants that were systemically infected (the virus spread from the inoculated leaf to other tissues and induced a systemic infection), and plants that were not hosts to the virus (Table 2). The symptoms produced in some of these hosts are shown in Figure 1. Note that, although tomato plants initially produced severe symptoms following

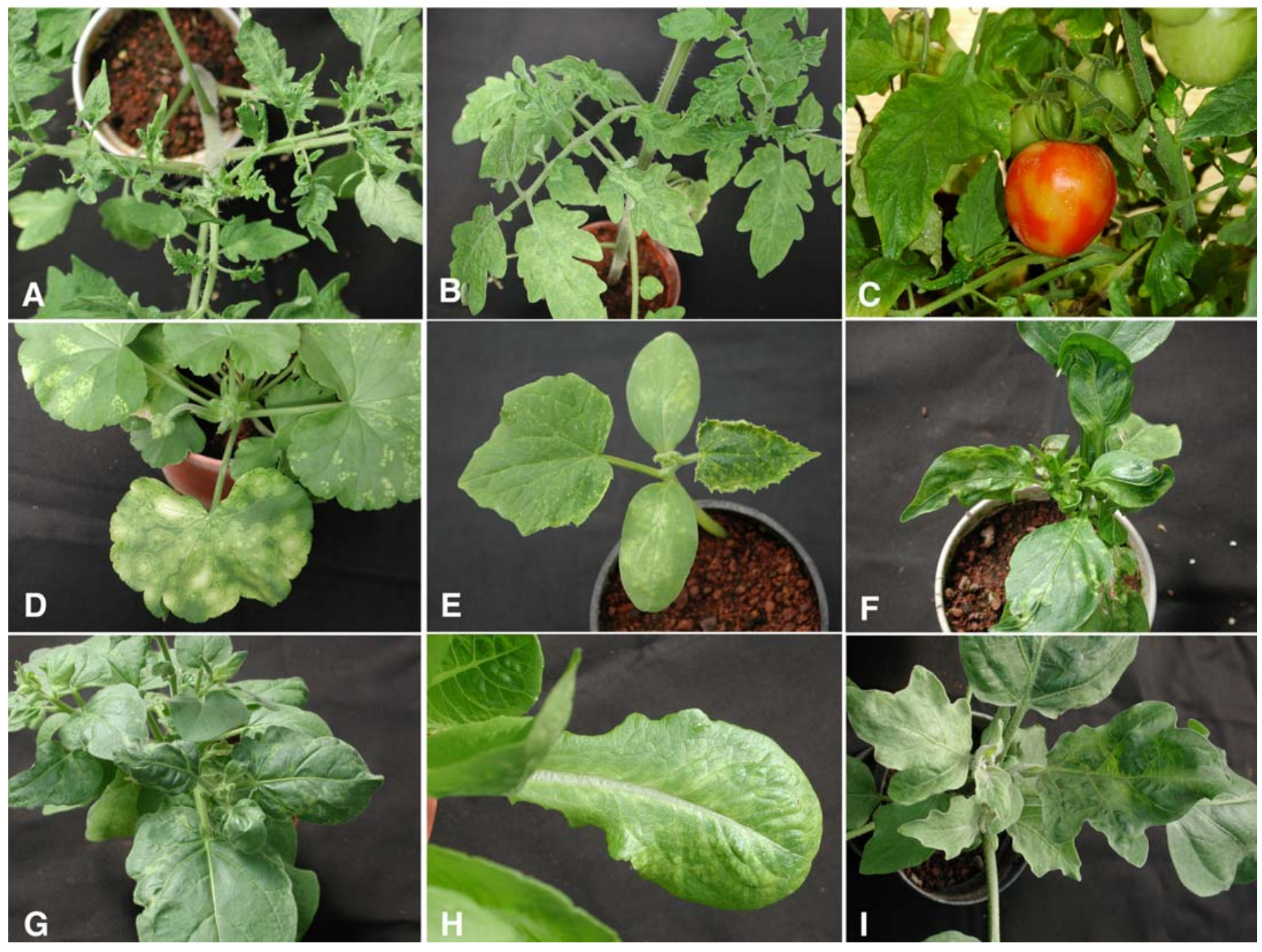

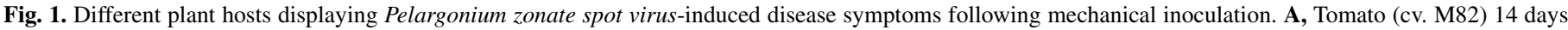

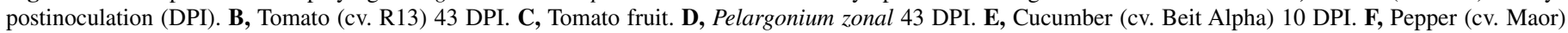
14 DPI. G, Nicotiana glutinosa 14 DPI. H, Lettuce 14 DPI. I, Eggplant 14 DPI. 
inoculation with PZSV, $\approx 3$ to 4 weeks later, the plants appeared to have undergone partial recovery: disease symptoms became milder, resembling the symptoms observed in the field, and the plants were able to flower and produce fruit. However, these fruit usually showed disease symptoms as well (Fig. 1).

All of the inoculated indicator plants were tested for systemic infection of PZSV by inoculation of $N$. glutinosa test plants with sap from the apex of the infected plants. None of the inoculated $N$. glutinosa test plants exhibited symptoms when sap was taken from the apex of plants with localized infected or nonhost plants but all of the systemically infected plants were positive when tested by inoculation to $N$. glutinosa. Plants with localized infection and nonhost plants were also tested for the presence of PZSV by RT-PCR, and the results confirmed those from the backinoculation tests.

Soil transmission. To test whether PZSV can be transmitted through contaminated soil (i.e., whether virus present in roots of infected plants can serve as an infection source), PZSV-infected $N$. glutinosa plants were grown for nearly 2 months and then removed while leaving their infected roots in the soil. Then, 14-day-old (first-true-leaf stage) tomato seedlings were transplanted into the contaminated soil and allowed to grow for another 2 months. In all, $\approx 50$ tomato seedlings were transplanted, none of which produced PZSV symptoms and all of which tested negative for the virus by back inoculation to $N$. glutinosa plants and by RT-PCR. Samples were taken from the infected $N$. glutinosa roots immediately after removal of the infected plants and a second time 10 days after removal of the infected plants. The infected roots samples were tested for the presence of PZSV by RT-PCR and by back inoculation to $N$. glutinosa test plants. All the samples were PZSV positive by RT-PCR, and the backinoculated $N$. glutinosa plants developed typical PZSV symptoms (not shown), thus demonstrating that the roots of infected $N$. glutinosa plants contain viable PZSV and that the virus remains infectious at least for 10 days after removal of the plants.

Seed transmission. Infected tomato plants (cvs. M82 and R13) were grown until fruiting and the seed were harvested, washed with water, and dried. They were then sown in Todd planter flats, grown for 1 month in the greenhouse, and then evaluated for the development of symptoms characteristic of PZSV. Nearly $19 \%$ of the seedlings grown from these seed developed disease symptoms (Table 3). The presence of PZSV in the symptomatic plants was confirmed by RT-PCR and by back inoculation to $N$. glutinosa plants (not shown). To eliminate the possibility that viral seed

TABLE 3. Effect of seed treatment on transmission of Pelargonium zonate spot virus

\begin{tabular}{llccc}
\hline & & \multicolumn{2}{c}{ No. of germinated seedlings } & \\
\cline { 3 - 4 } Tomato cv. & Treatment & Infected & Total & Infection rate (\%) \\
\hline M82 & Water & 121 & 646 & 18.7 \\
R13 & Water & 19 & 136 & 13.9 \\
& HCl+TSP & 17 & 154 & 11.0 \\
\hline
\end{tabular}

a $\mathrm{TSP}=$ trisodium phosphate.

TABLE 1. Comparison of nucleotide and amino acid identity of predicted open reading frames (ORFs) between the genomic components of Pelargonium zonate spot virus (PZSV) from Israel and PZSV from Italy

\begin{tabular}{|c|c|c|c|c|c|c|}
\hline \multirow[b]{2}{*}{ ORF } & \multicolumn{4}{|c|}{ PZSV-Israel } & \multicolumn{2}{|c|}{ PZSV-Italy } \\
\hline & Start & Stop & Nucleotide identity $(\%)^{\mathrm{a}}$ & Amino acid identity $(\%)^{\mathrm{a}}$ & Start & Stop \\
\hline RNA 1a & 79 & 2,943 & 90 & 93 & 79 & 2,967 \\
\hline RNA $2 \mathrm{a}$ & 82 & 2,145 & 95 & 97 & 82 & 2,145 \\
\hline RNA 3a & 335 & 1,264 & 97 & 98 & 335 & 1,264 \\
\hline RNA $3 b$ & 1,618 & 2,244 & 92 & 96 & 1,619 & 2,245 \\
\hline
\end{tabular}

${ }^{a}$ Percent nucleotide and amino acid identity with PZSV from Italy.

TABLE 2. Experimental host range of Pelargonium zonate spot virus (PZSV)-Israel ${ }^{\mathrm{a}}$



${ }^{a}$ PZSV-infected Nicotiana glutinosa plants were used to sap inoculate test plants, as described in Materials and Methods. At least 5 plants of each accession were inoculated.

${ }^{\mathrm{b}}$ Disease symptoms: None $=$ no symptoms following inoculation, Systemic $=$ symptoms appeared at plant apex, $\mathrm{Cl}=\mathrm{chlorotic}$ lesions, $\mathrm{Nl}=\mathrm{necrotic}$ lesions, $\mathrm{Mo}=$ mosaic, $\mathrm{De}=$ deformation, $\mathrm{Le}=$ leaf elongation, $\mathrm{Yr}=$ yellow rings, $\mathrm{St}=$ stunting.

${ }^{\mathrm{c}}$ Samples were taken from the apex of the infected plants and infectivity (+) or lack of (-) was determined by back-inoculation to $N$. glutinosa test plants as described in Materials and Methods.

d Samples were taken from the apex of the infected plants and presence $(+)$ or absence $(-)$ of PZSV was determined by RT-PCR. ND $=$ not determined. 
transmission was due to virus present on the outer surface of the seed, the seed were treated either with only water or with $2 \% \mathrm{HCl}$ followed by $10 \%$ TSP. Seedlings grown from water-treated seed and those grown from seed following $\mathrm{HCl}$ and TSP treatment had approximately similar PZSV infection rates (Table 3).

Seed from an infected tomato plant were also tested for the presence of PZSV by RT-PCR: the virus was detected in total RNA extracted from as few as 5 seeds (Fig. 2). Moreover, because these seed were not destined for germination, it was possible to subject them to a much harsher disinfection treatment $(10 \% \mathrm{HCl}$ and $10 \%$ TSP). Even with this treatment, the seed remained positive for PZSV by RT-PCR (Fig. 2), as they did after additional treatments with sulfuric acid (not shown) or bleach (Fig. 2).

Pollen transmission. Pollen was collected from flowers of infected tomato plants; total nucleic acids were extracted and subjected to RT-PCR with PZSV-specific primers. Indeed, the pollen was found to contain PZSV (Fig. 3). Hence, to determine whether the virus was pollen transmitted, pollen was collected from flowers of infected plants and flowers of healthy mother plants were hand pollinated. None of the hand-pollinated mother plants became infected with PZSV. Seed collected from those plants and treated with $2 \% \mathrm{HCl}+10 \%$ TSP were sown, and the germinated seedlings were monitored for the appearance of PZSV symptoms. Nearly $30 \%$ of the germinated seedlings showed clear disease symptoms (Table 4). The symptomatic seedlings were also tested by back inoculation, and all of them were found to be infected with PZSV.

We also looked at the variability of seed transmission of PZSV by comparing seed produced from individual fruit from the same plant following hand pollination. Results from plants that produced five or more fruit following hand pollination of healthy mother plants with pollen from infected plants (the same plants as in Table 4) are shown in Table 5. The transmission rate varied from 0 to $10 \%$ at the lower end to 50 to $88 \%$ at the higher end. However, these values appeared to be the exception to the rulein the vast majority of the fruit, seed transmission was 20 to $35 \%$ (Table 5).

\section{DISCUSSION}

The causal agent of a new tomato disease in Israel was identified as a new isolate of PZSV. The genome of this PZSV isolate identified in Israel showed very high nucleotide sequence identity with the isolate that was previously identified and cloned in Italy. The virus was readily transmitted mechanically and induced symptoms resembling those described previously (5). The host range was essentially similar between the different PZSV isolates, with the major differences being (i) the isolates from Israel, France, and the United States infect $N$. benthamiana whereas the Italian "type-isolate" does not; (ii) the isolates from Israel, Spain, and France all infect Datura stramonium whereas the Italian isolate does not; (iii) the reaction of cucumber plants to PZSV was not clear-the Italian isolate was reported to induce local lesions followed by systemic infection whereas, in France and Spain, cucumber has been reported as a local-lesion-type host (we found the reaction of cucumber plants to PZSV to be cultivar-

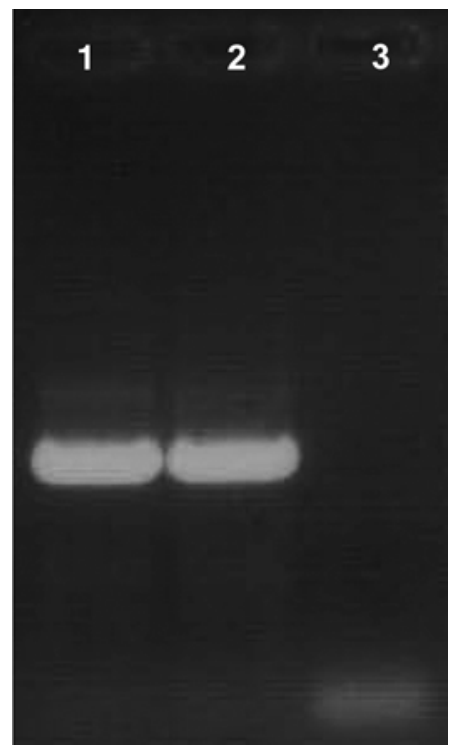

Fig. 3. Reverse-transcription polymerase chain reaction (RT-PCR) identification of Pelargonium zonate spot virus (PZSV) RNA in pollen collected from flowers of PZSV-infected tomato plants. Total RNA was extracted from the pollen grains and subjected to RT-PCR with PZSV-specific primers (see Materials and Methods). Lanes 1 and 2: pollen from infected plants. Lane 3: pollen from a healthy plant.

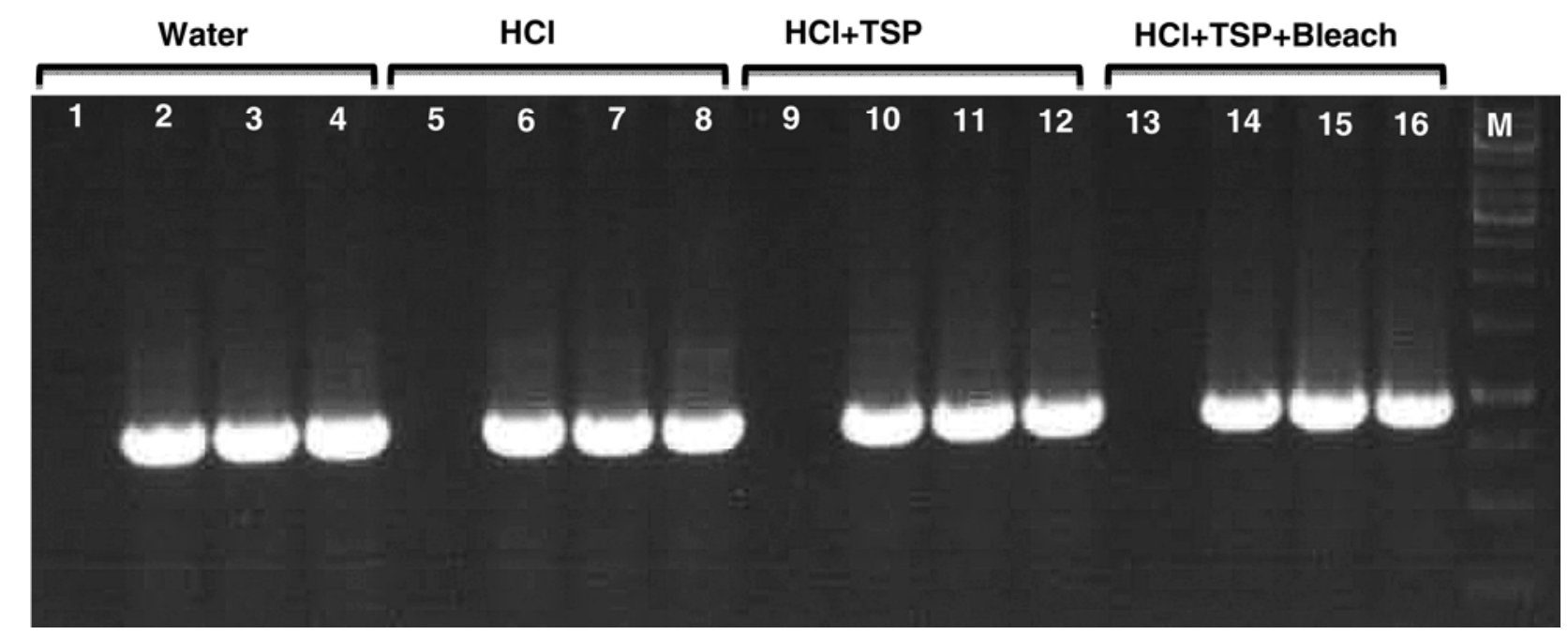

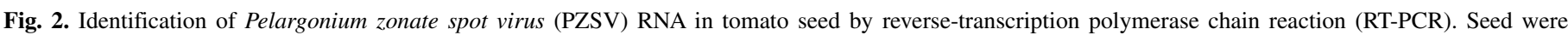

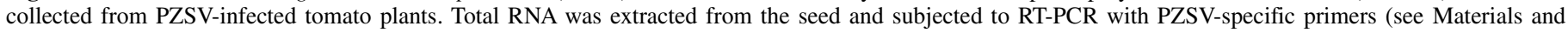

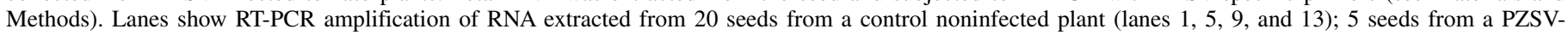

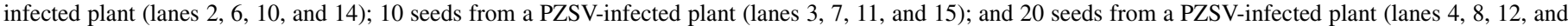

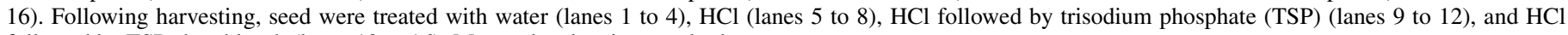
followed by TSP, then bleach (lanes 13 to 16 ). $\mathrm{M}=$ molecular size standards. 
dependent—whereas cv. Shimshon was a nonhost to the virus, cv. Beit Alpha was a systemic host); and (iv) our finding of lettuce and eggplant as PZSV hosts has never been reported. These differences can be explained by the fact that different cultivars were tested, under different conditions, or by the possibility that the virus has undergone some minor changes since it was first isolated and characterized in Italy; the study in Italy was done in 1982, 27 years ago, whereas the other strains have been isolated and characterized within the last 10 years.

This is the first identification of PZSV in Israel but it has also been detected recently in California (13). This led us to wonder how this virus, which does not appear to have an insect vector, has begun spreading to different and distant geographical regions. Hence, we looked for ways other than mechanical transmission for the virus to spread. Indeed, when we collected seed from infected tomato plants, they were found to contain PZSV by RTPCR, and the virus was able to infect the seedlings that developed from these infected seed. Seed-disinfectant treatments have been shown to remove viruses that adhere to the seed surface; TSP treatment of tomato seed has been used successfully for many years to eliminate Tomato mosaic virus (ToMV) (8). ToMV is carried on the seed surface, while seedling infection occurs mainly by mechanical transmission through wounds that are incurred when the seedlings are being handled $(1,8,16,19)$. Recently, it has been shown that disinfection of tomato seed with TSP can also eliminate Pepino mosaic virus seed transmission (2). However, regardless of the seed-disinfectant treatment used, PZSV remained detectable in seed by RT-PCR. Even when testing as few as 5 seeds, the virus was easily detectable. Moreover as already mentioned, when the PZSV-infected seed were sown, the virus was transmitted to the newly emerging seedlings. PZSV transmission rate from seed to seedling was 11 to $29 \%$, regardless of the seed-disinfection treatment. These results strongly suggest that PZSV is transmitted vertically within the infected seed and not on its surface.

Once we found that PZSV can be transmitted through the seed in tomato, pollen was collected from flowers of a PZSV-infected plant and tested for presence of the virus. Using RT-PCR, we found that pollen grains do harbor the virus. We then tested whether pollen grains can transmit the virus by hand pollinating noninfected mother plants with pollen grains collected from PZSV-infected plants. We hand pollinated more than 60 flowers from 13 different mother plants. None of the mother plants became infected with PZSV (no horizontal transmission by infected pollen) but the seed resulting from these crosses transmitted the virus at an efficiency of $29 \%$. Hence, in tomato, PZSV is transmitted vertically by pollen as well.

TABLE 4. Seed transmission of Pelargonium zonate spot virus following hand pollination of healthy mother plants with pollen collected from infected tomato plants

\begin{tabular}{lcccc}
\hline & & \multicolumn{2}{c}{ No. of germinated seedlings } & \\
\cline { 3 - 4 } Plant no. & No. of fruit & Infected & Total & Infection rate (\%) \\
\hline 1 & 1 & 3 & 9 & 33.3 \\
2 & 7 & 30 & 75 & 40.0 \\
3 & 4 & 15 & 57 & 26.3 \\
4 & 9 & 83 & 280 & 29.6 \\
5 & 10 & 35 & 120 & 29.2 \\
6 & 4 & 37 & 131 & 28.2 \\
7 & 4 & 25 & 98 & 25.5 \\
8 & 4 & 14 & 56 & 25.0 \\
9 & 7 & 74 & 270 & 27.4 \\
10 & 2 & 12 & 28 & 42.9 \\
11 & 4 & 22 & 86 & 25.6 \\
12 & 5 & 41 & 165 & 24.8 \\
13 & 2 & 13 & 79 & 16.5 \\
Total & $\ldots$ & 404 & 1,454 & $\ldots$ \\
Average & $\ldots$ & $\ldots$ & $\ldots$ & 28.8 \\
\hline
\end{tabular}

PZSV has been shown to be seed-transmitted in $N$. glutinosa and Diplotaxis erucoides, both with an efficiency of $\approx 5 \%(5,20)$. Vovlas et al. (21) found that pollen collected from infected tomato plants is contaminated with PZSV but that the virus was not transmitted by tomato seed. Our results, demonstrating that PZSV is transmitted by both pollen and seed in tomato, are clearly different. However, seed transmission of a plant virus is affected by a number of biological parameters, which can account for the differences in the various reports. It has been shown that different viral isolates vary in their ability to be seed transmitted, that different cultivars of the same host species vary in their ability to seed-transmit a virus, and that the efficiency of seed transmission is influenced by environmental conditions $(10,15,16)$. It should be noted that PZSV was recently assigned to the new genus Anulavirus, within the family Bromoviridae (6). Thus far, this genus contains a single virus: PZSV. Only two PZSV isolates were fully cloned and sequenced-the Italian isolate and the Israeli isolate reported here. The major difference between the two isolates is that PZSV-Il is seed and pollen transmitted in tomato whereas PZSV-It is not. This new mode of transmission has clear epidemiological consequences, and may explain the recent spread of PZSV noted in Israel, the United States, and Spain $(3,13)$.

Tomato is a valuable crop in many regions in the world. Obviously, pollen and seed transmission of PZSV pose a major concern for the tomato industry. Most commercial hybrid tomato seed are grown and harvested in one geographical site but marketed to others. For instance, most hybrid tomato seed sold in Europe are produced elsewhere, usually in countries where production costs are low, such as China, Thailand, India, Chile, and so on. Thus, even low rates of viral seed transmission can facilitate the introduction of viruses into new geographical and previously noninfected tomato-production areas. The concern is even greater when the virus is also pollen transmitted. To protect precious hybrids, many seed companies grow their hybrid parents in different sites, sometimes even in different countries. Pollen is

TABLE 5. Variability in seed transmission among fruit of the same plant following hand pollination of healthy mother plants with pollen collected from infected tomato plants

\begin{tabular}{|c|c|c|c|c|}
\hline \multirow[b]{2}{*}{ Plant no. } & \multirow[b]{2}{*}{ Fruit no. } & \multicolumn{2}{|c|}{ No. of germinated seedlings } & \multirow[b]{2}{*}{ Infection rate $(\%)$} \\
\hline & & Infected & Total & \\
\hline \multirow[t]{9}{*}{$\overline{4}$} & 1 & 15 & 47 & 32 \\
\hline & 2 & 3 & 8 & 38 \\
\hline & 3 & 21 & 64 & 33 \\
\hline & 4 & 5 & 13 & 38 \\
\hline & 5 & 6 & 19 & 32 \\
\hline & 6 & 16 & 58 & 28 \\
\hline & 7 & 4 & 19 & 21 \\
\hline & 8 & 6 & 17 & 35 \\
\hline & 9 & 7 & 35 & 20 \\
\hline \multirow[t]{8}{*}{5} & 1 & 7 & 23 & 30 \\
\hline & 2 & 6 & 19 & 32 \\
\hline & 3 & 5 & 17 & 29 \\
\hline & 4 & 3 & 11 & 27 \\
\hline & 5 & 1 & 10 & 10 \\
\hline & 6 & 3 & 17 & 29 \\
\hline & 7 & 3 & 10 & 30 \\
\hline & 8 & 7 & 8 & 88 \\
\hline \multirow[t]{6}{*}{9} & 1 & 7 & 35 & 20 \\
\hline & 2 & 15 & 44 & 34 \\
\hline & 3 & 19 & 55 & 35 \\
\hline & 4 & 19 & 75 & 25 \\
\hline & 5 & 11 & 51 & 22 \\
\hline & 6 & 3 & 6 & 50 \\
\hline \multirow[t]{5}{*}{12} & 1 & 27 & 89 & 30 \\
\hline & 2 & 3 & 12 & 25 \\
\hline & 3 & 7 & 32 & 22 \\
\hline & 4 & 0 & 18 & 0 \\
\hline & 5 & 4 & 14 & 29 \\
\hline
\end{tabular}


collected in the field from the male parent and transported to the field of the mother plants, which are hand pollinated. This may increase the risk of spread of a pollen-transmitted virus such as PZSV, particularly because it appears that tomato plants show some recovery from PZSV-induced symptoms (Fig. 1); these much milder symptoms may be overlooked under field conditions. Hence, pollen collectors may fail to notice that pollen is being collected from an infected plant.

The commercial use of insect pollinators, such as bumblebees, is widespread in many tomato-production greenhouses worldwide. This practice further increases the danger of PZSV spread from pollen of infected plants to seedlings of healthy ones. Moreover, pollinating insects can provide a means for dispersing the virus over long distances (hundred of miles), as has been reported before for other pollen-transmitted viruses (16).

\section{ACKNOWLEDGMENTS}

Contribution from the Agricultural Research Organization, Volcani Center, Bet Dagan, Israel. Number 131/2009. We thank J. Polston (University of Florida) for critically reviewing this manuscript.

\section{LITERATURE CITED}

1. Broadbent, L. 1965. The epidemiology of tomato mosaic. XI. Seedtransmission of TMV. Ann. Appl. Biol. 56:177-205.

2. Cordoba-Selles, M. C., Garcia-Randez, A., Alfrado-Fernandez, A., and Jorda-Gutierrez, C. 2007. Seed transmission of Pepino mosaic virus and efficacy of tomato seed disinfection treatments. Plant Dis. 91:12501254.

3. Escriu, F., Cambra, M. A., and Luis-Artega, M. 2009. First report of pepper as a natural host for Pelargonium zonate spot virus in Spain. Plant Dis. 93:1346.

4. Finetti-Sialer, M., and Gallitelli, D. 2003. Complete nucleotide sequence of Pelargonium zonate spot virus and its relationship with the family Bromoviridae. J. Gen. Virol. 84:3143-3151.

5. Gallitelli, D. 1982. Properties of the tomato isolate of Pelargonium zonate spot virus. Ann. Appl. Biol. 100:457-466.

6. Gallittelli, D., Finetti-Sialer, M., and Martelli, G. P. 2005. Anulavirus, a proposed new genus of plant viruses in the family Bromoviridae. Arch. Virol. 150:407-411.

7. Gebre-Selassie, K., Dellecolle, B., Gognalons, P., Dufour, O., Gros, C., Cotillon, A. C., Parrella, G., and Marchoux, G. 2002. First report of an isolate of Pelargonium zonate spot virus in commercial glasshouse tomato crop in Southeastern France. Plant Dis. 86:1052.

8. Green, S. K., Hwang, L. L., and Kuo, Y. K. 1987. Epidemiology of tomato mosaic virus in Taiwan and identification of strains. J. Plant Dis. Prot. 94:386-387.

9. Gulati-Sakhuja, A., Sears, J. L., Nunez, A., and Liu, H.-Y. 2009. Production of polyclonal antibodies against Pelargonium zonate spot virus coat protein expressed in Escherichia coli and application for immunodiagnosis. J. Virol. Methods 160:29-37.

10. Johansen, E., Edwards, M. C., and Hampton, R. O. 1994. Seed transmission of viruses: Current perspectives. Annu. Rev. Phytopathol. 32:363386.

11. Lapidot, M., Paran, I., BenJoseph, R., BenHarush, S., Pilowsky, M., Cohen, S., and Shifriss, C. 1997. Tolerance to cucumber mosaic virus in pepper: development of advanced breeding lines and evaluation of virus level. Plant Dis. 81:185-188.

12. Levin, I., and Gilboa, N. 1997. Direct PCR using tomato pollen grain suspension. BioTechniques 23:986-990.

13. Liu, H.-Y., and Sears, J. L. 2007. First report of Pelargonium zonate spot virus from tomato in the United States. Plant Dis. 91:633.

14. Luis-Arteaga, M., and Cambra, M. 2000. First report of natural infection of greenhouse-grown tomato and weed species by Pelargonium zonate spot virus in Spain. Plant Dis. 91:633.

15. Maule, A. J., and Wang, D. 1996. Seed transmission of plant viruses: A lesson in biological complexity. Trends Microbiol. 4:153-158

16. Mink, G. I. 1993. Pollen- and seed-transmitted viruses and viroids. Annu. Rev. Phytopathol. 31:357-402.

17. Quacquarelli, A., and Gallitelli, D. 1979. Tre virosi del geranio in Puglia. Phytopathol. Mediterr. 19:61-70.

18. Roossinck, M. J., and White, P. S. 1998. Cucumovirus isolation and RNA extraction. Pages 189-196 in: Methods in Molecular Biology, Plant Virology Protocols from Virus Isolation to Transgenic Resistance, Vol. 81. G. D. Foster and S. C. Taylor, eds. Humana Press Inc., Totowa, NJ.

19. Taylor, R. H., Grogan, R. G., and Kimble, K. A. 1961. Transmission of tobacco mosaic virus in tomato seed. Phytopathology 51:837-842.

20. Vovlas, C., Gallitelli, D., and Conti, M. 1989. Preliminary evidence for an unusual mode of transmission in the ecology of Pelargonium zonate spot virus (PZSV). Pages 302-305 in: 4th Plant Virus Epidemiol. Workshop. Montpellier, France. 